\title{
Petroleum Pumps' Current and Vibration Signatures Analysis Using Wavelet Coherence Technique
}

\author{
Rmdan Shnibha and Alhussein Albarbar \\ Advanced Industrial Diagnostic Centre, Digital Signal Processing Research Group, School of Engineering, Manchester Metropolitan \\ University, Manchester M1 5GD, UK
}

Correspondence should be addressed to Alhussein Albarbar; a.albarbar@mmu.ac.uk

Received 28 February 2013; Revised 7 May 2013; Accepted 22 May 2013

Academic Editor: K. M. Liew

Copyright (C) 2013 R. Shnibha and A. Albarbar. This is an open access article distributed under the Creative Commons Attribution License, which permits unrestricted use, distribution, and reproduction in any medium, provided the original work is properly cited.

\begin{abstract}
Vibration analysis is widely used for rotating machinery diagnostics; however measuring vibration of operational oil well pumps is not possible. The pump's driver's current signatures may provide condition-related information without the need for an access to the pump itself. This paper investigates the degree of relationship between the pump's driver's current signatures and its induced vibration. This relationship between the driver's current signatures (DCS) and its vibration signatures (DVS) is studied by calculating magnitude-squared coherence and phase coherence parameters at a certain frequency band using continuous wavelet transform (CWT). The CWT coherence-based technique allows better analysis of temporal evolution of the frequency content of dynamic signals and areas in the time-frequency plane where the two signals exhibit common power or consistent phase behaviour indicating a relationship between the signals. This novel approach is validated by experimental data acquired from $3 \mathrm{~kW}$ petroleum pump's driver. Both vibration and current signatures were acquired under different speed and load conditions. The outcomes of this research suggest the use of DCS analysis as reliable and inexpensive condition monitoring tool, which could be implemented for oil pumps, real-time monitoring associated with condition-based maintenance $(\mathrm{CBM})$ program.
\end{abstract}

\section{Introduction}

Pumps and their associated systems are essential in oil and gas facilities for the efficient transportation of fluids. Common pumps found in these facilities include centrifugal, reciprocating, diaphragm, and rotary pumps [1]. The condition monitoring of pumps and their associated systems is an established application of CBM and is an existing area of research [2]. Rohlfing [3] provides three examples in the oil and gas industry where pump's CBM has been effectively implemented. Azadeh et al. in [4] have developed a diagnostic mechanism for pump failures in which pump operating problems fall into two categories: (1) hydraulic problems that suggest the pump may fail to deliver liquid, deliver insufficient capacity, develop insufficient pressure, or lose its prime at starting and (2) mechanical problems that are characterised by the consumption of excessive power or development of mechanical difficulties at the seal chambers or bearings; in either case vibration, noise, or breakage may occur. Fatigue is a common cause of pump failure $[5,6]$.
Vibration monitoring is particularly suited to pumps due to the number of integrated rotating parts, which may show additional movement when faults develop [7]. A more recent development in pump condition monitoring is the application of ultrasonic sensors [8]; introducing a new ultrasonic measurement based on acoustic emission analyses for highpressure process pumps.

The most obvious technique for obtaining a vibration signal from pump driver's "induction motor" is by direct measurement using vibration transducers (usually accelerometers) mounted on the driver. This requires a high-performance vibration transducer capable of withstanding harsh environmental conditions and which can cost several hundred dollars. When a large number of machines are concerned and more than one transducer is required per machine, the total cost can be high. A major disadvantage of vibration monitoring is that it requires access to the machine. For accurate measurements, sensors should be mounted rigidly on the machine, which requires expertise and trained personnel. 
An increasing number of pumps are installed with electrical motors as their prime driver. This development has introduced new possibilities for condition monitoring by the use of driver's electric signals such as current and voltage.

In response an indirect method, called sensorless detection and diagnosis intended for mechanical equipment driven by AC induction motors, has been developed over the past 20 years and is growing rapidly [9]. Sensorless detection and diagnosis technology monitors the state of devices and motors using the motor's stator current rather than external sensors to detect vibration, noise, and so forth. Besides the original power frequency, the stator current contains a large amount of information relative to mechanical faults.

The relationship between the magnitudes of stator current harmonics, the magnitudes of the vibration harmonics, and specific machine faults types has been closely studied $[9,10]$. In [9] the relationship between the vibration and current harmonic magnitudes for a source of known vibration frequency was investigated to determine the feasibility of setting a limit or standard on the current harmonics due to these vibrations. It was concluded that for a given vibration frequency, the harmonic's RMS vibration level and RMS current level are monotonically related. In [10] a method for sensorless on-line vibration monitoring of an induction motor was proposed and initially evaluated in the laboratory and it was shown that vibration information can be gained in a sensorless fashion by utilizing the nearly linear relation between a particular vibration spectral component and its corresponding current harmonics. This forms the basis for using the current harmonics as an indicator of motor vibrations. The constant of proportionality between the current and vibration harmonics is obtained by measuring a baseline value of vibration. Baseline measurements were taken for the normal operation condition of the test machine, which included a small externally induced vibration. Experimental results clearly showed that this method is applicable for a wide range of vibrations.

The cause and effect relationship between two signals or the commonality between them is generally estimated using the coherence function. Reference [11] presents the results of a case study of motor bearing degradation caused by accelerated electrical discharge machining under the seven aging cycles. To identify bearing damage using the motor current signal, the coherence function between the motor current and vibration signature was computed. The largest values of the coherence amplitude, when driver's current and vibration signals were correlated, were located at the dynamic eccentricity and bearing defect.

This paper aims at investigating the use of current signal as reliable and inexpensive tool for pump's CBM programs. It is organised as follows. In Section 2 wavelet coherence is presented. Some experimental data for validating this approach are presented and some results are given. Concluding remarks appear in Section 3.

\section{Coherence Analysis Technique}

Figure 1 shows the time domain of current and vibration signals for a healthy motor and motor with a $20 \mathrm{~V}$ drop in one phase of the supply. Figure 2 shows the RMS values of current and vibration for different motor loads; it can be seen that the stator current and vibration signals are inversely correlated.

A common approach for extracting information concerning frequency features of a periodic signal is to transform the signal to the frequency domain using the discrete Fourier transform.

In Figure 3, the same signals as those shown in Figure 1 are presented in the frequency domain; it is assumed that the original signals are stationary.

The coherence is a function of the power spectral density $\left(P_{x x}\right.$ and $\left.P_{y y}\right)$ of $x$ and $y$ and the cross power spectral density $\left(P_{x y}\right)$ of $x$ and $y$ is given by

$$
C_{x y}(f)=\frac{\left|P_{x y}(f)\right|^{2}}{P_{x x}(f) P_{y y}(f)} .
$$

Coherence is a function of frequency with $C_{x y}(f)$ ranging between 0 and 1 and indicates how well signal $x$ corresponds to signal $y$ at each frequency. The degree of synchronization in stator current signal and vibration signal is commonly characterized by coherence phase and magnitude-squared coherence (DSC).

2.1. Welch's Method. An improved estimator of the PSD is the one proposed by Welch [8]. The method consists of dividing the time series data into (possibly overlapping) segments, computing a modified periodogram of each segment and then averaging the PSD estimates. The result is Welch's PSD estimate.

The averaging of modified periodograms tends to decrease the variance of the estimate relative to a single periodogram estimate of the entire data record. Although overlap between segments tends to introduce redundant information, this effect is diminished by the use of a nonrectangular window, which reduces the importance or weight given to the end samples of segments (the samples that overlap).

However, as mentioned above, the combined use of short data records and nonrectangular windows results in reduced resolution of the estimator. In summary, there is a trade-off between variance reduction and resolution. One can manipulate the parameters in Welch's method to obtain improved estimates relative to the periodogram, especially when the SNR is low.

The coherence function between the motor current and vibration signature was computed and plotted in Figure 4. The largest coherence amplitude values, where motor current and vibration signals are best correlated, are located at 50 and $100 \mathrm{~Hz}$.

2.2. Wavelet-Based Approach. The CWT allows analysis of the temporal evolution of the frequency content of a given signal or time series. The application of the CWT to two time series and the cross-examination of the two decompositions can reveal localized similarities in time and scale. Areas in the time-frequency plane where the two time series exhibit common power or consistent phase behaviour indicate a relationship between the signals. 


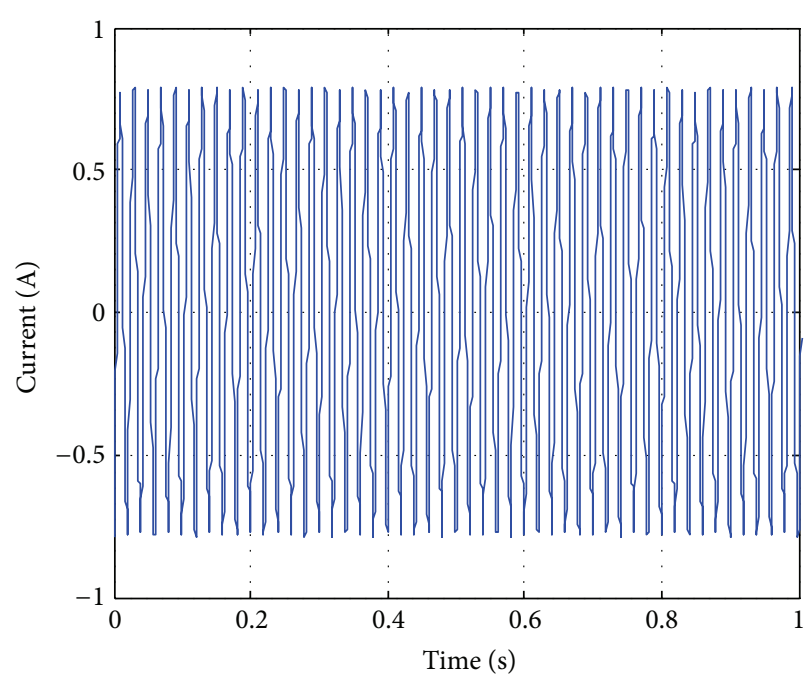

(a) Healthy motor with symmetrical supply voltages

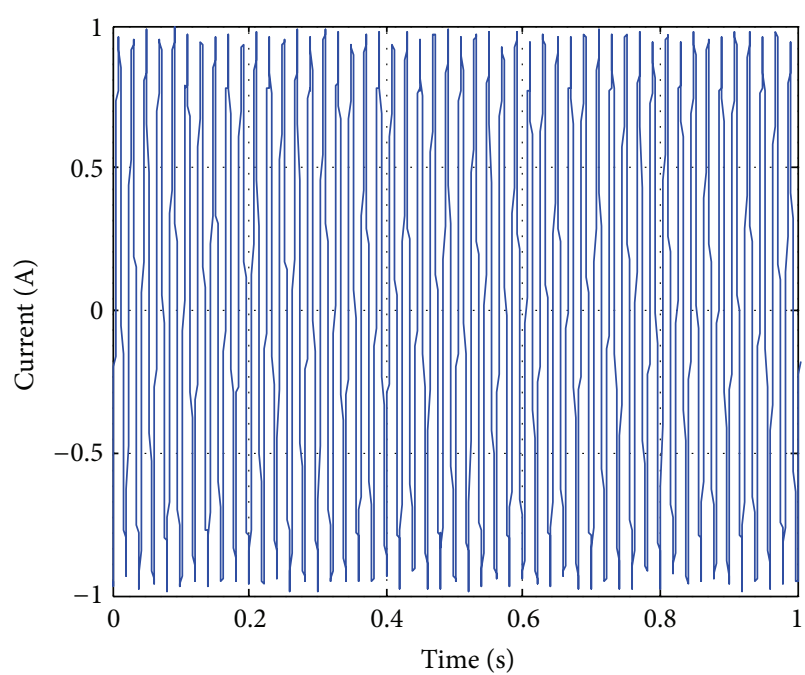

(c) One-phase supply voltage with 20 volts drop

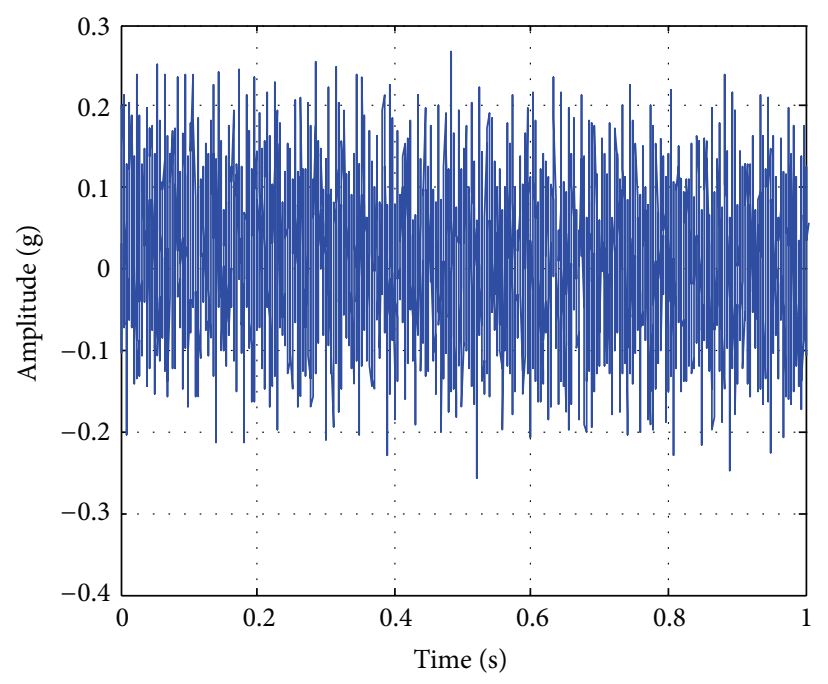

(b) Healthy motor with symmetrical supply voltages

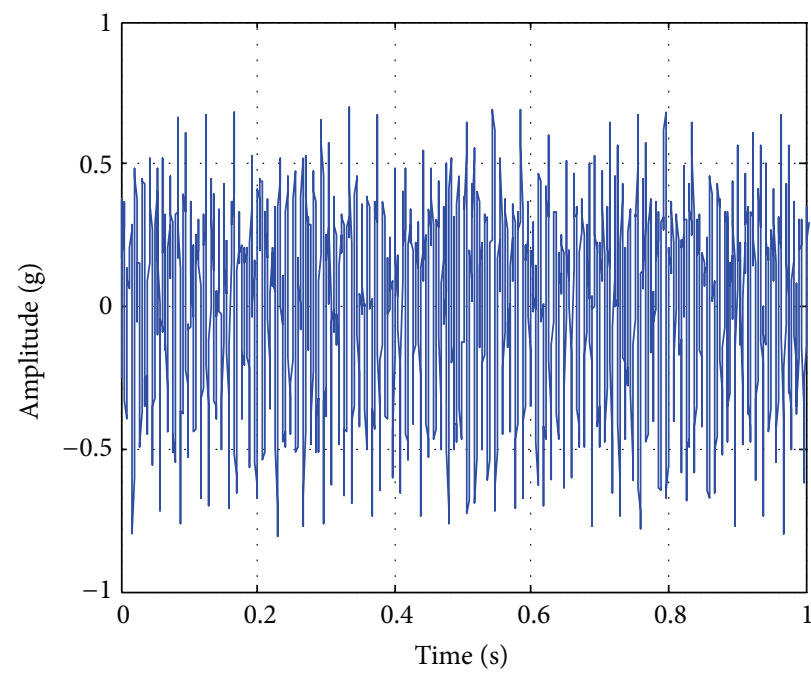

(d) One-phase supply voltage with 20 volts drop

FIgURE 1: Time waveforms of current and vibration signatures.

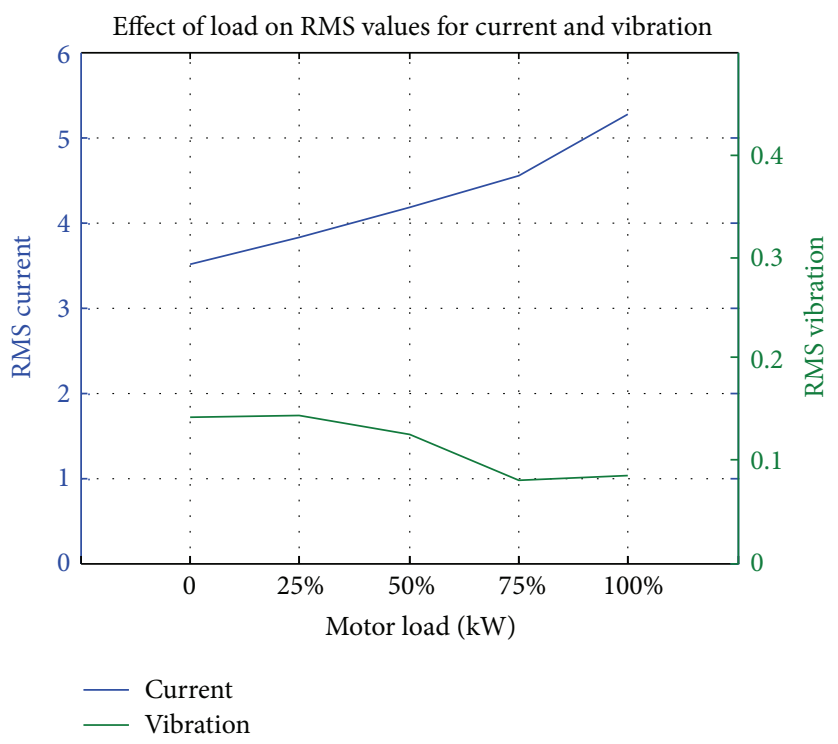

FIGURE 2: Influence of load on the RMS current and vibration signals. 


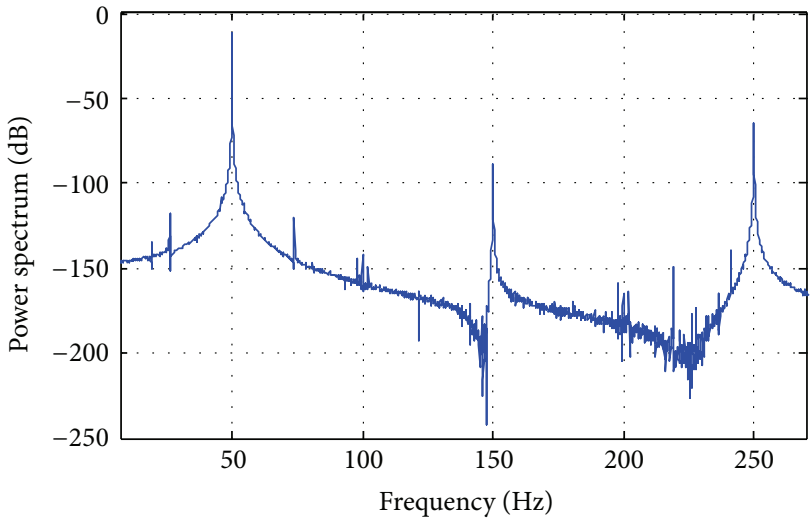

(a) Healthy motor with symmetrical supply voltages (current)

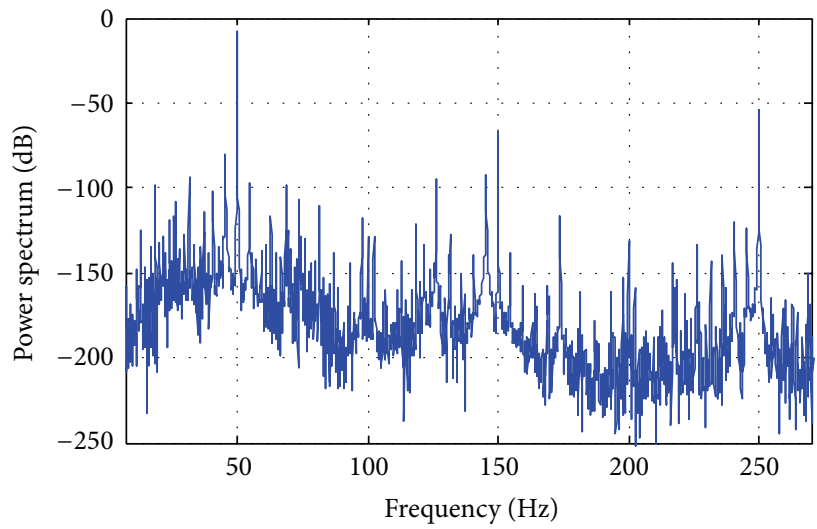

(c) One-phase supply voltage with 20 volts drop (current)

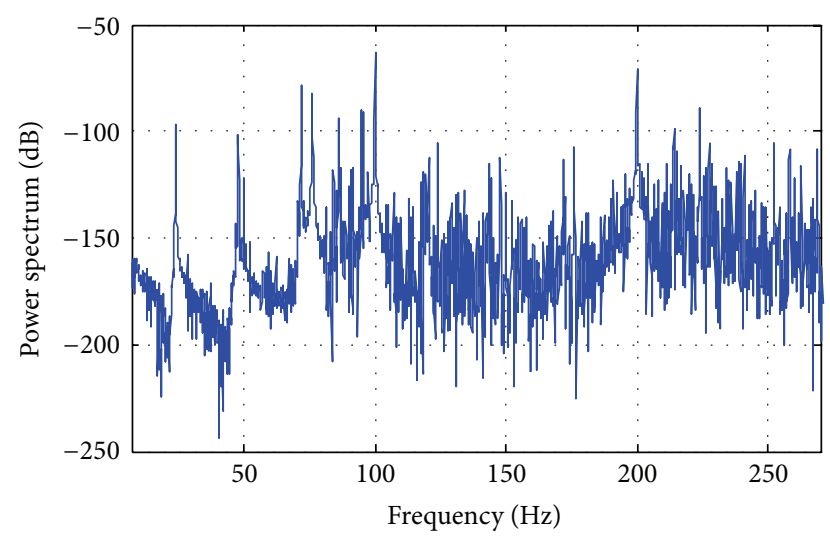

(b) Healthy motor with symmetrical supply voltages (vibration)

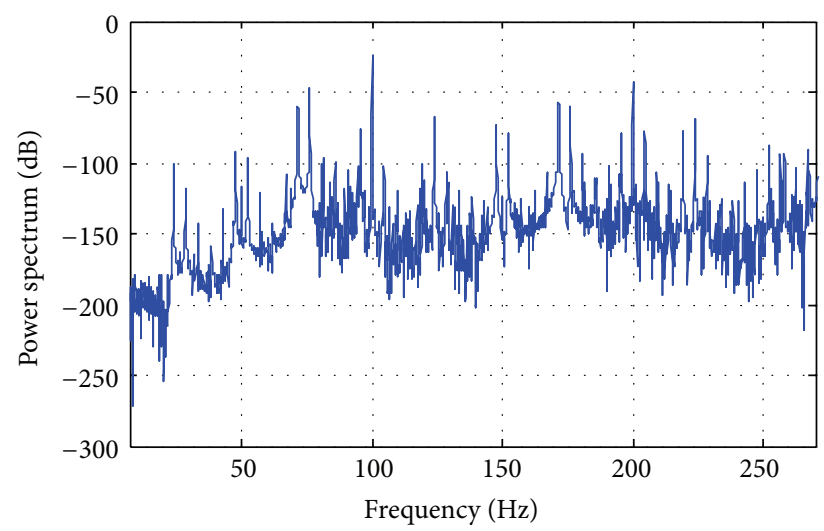

(d) One-phase supply voltage with 20 volts drop (vibration)

FIGURE 3: Frequency spectra of current and vibration signatures.

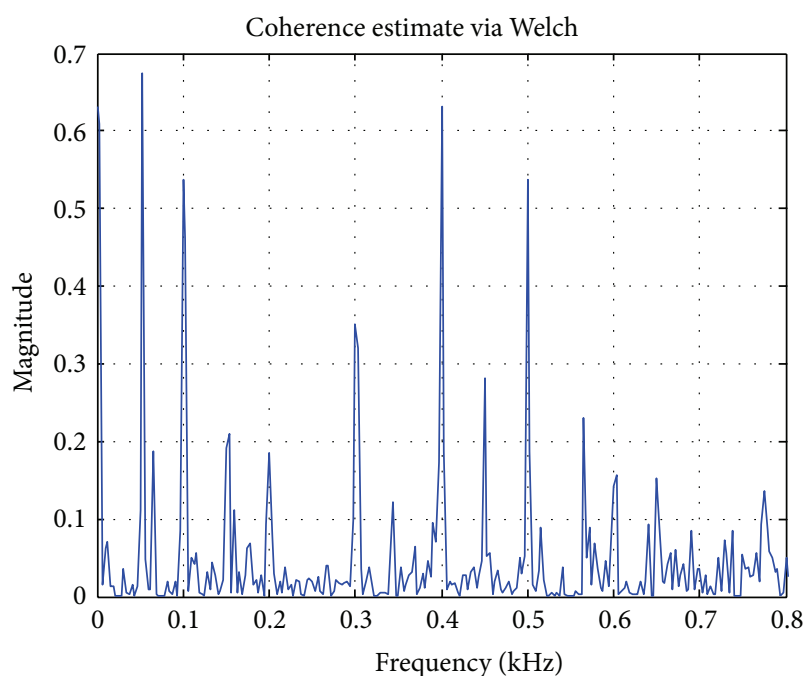

FIGURE 4: Coherence between DCS and DVS signals.

For jointly stationary time series, the cross spectrum and associated coherence function based on the Fourier transform are used to detect common behaviour in the frequency domain. In the general nonstationary case, wavelet-based counterparts can be defined to provide time-localized alternatives.
The wavelet coherence of two time series $x$ and $y$ is

$$
\frac{S\left[C_{x}^{*}(a, b) C_{y}(a, b)\right]}{\sqrt{S\left(\left|C_{x}(a, b)\right|^{2}\right)} \sqrt{S\left(\left|C_{y}(a, b)\right|^{2}\right)}}
$$

where $C_{x}(a, b)$ and $C_{y}(a, b)$ denote the continuous wavelet transforms of $x$ and $y$ at scale $a$ and position $b$. The superscript $*$ is the complex conjugate and $S$ is a smoothing operator in time and scale.

Figure 5 presents the CWT analysis of the current and vibration signals when a $3 \mathrm{~kW}$ driver running at healthy condition. The top left figure shown the CWT modulus of current signal and the top right figure shown the cwt modulus of vibration signal.

The common periods of the current and vibration signals at scales 32 and 16, respectively are clearly detected in the moduli of the individual wavelet spectra and frequency at 50 and $100 \mathrm{~Hz}$ as shown in Figure 5.

The wavelet spectrum, defined for each signal, is characterized by the modulus and the phase of the CWT obtained using the complex valued wavelet. The individual wavelet spectra are denoted as $C_{x}(a, b)$ and $C_{y}(a, b)$. The two decompositions are the same, up to a translation, since the CWT is translation-invariant. To examine the relationship between 


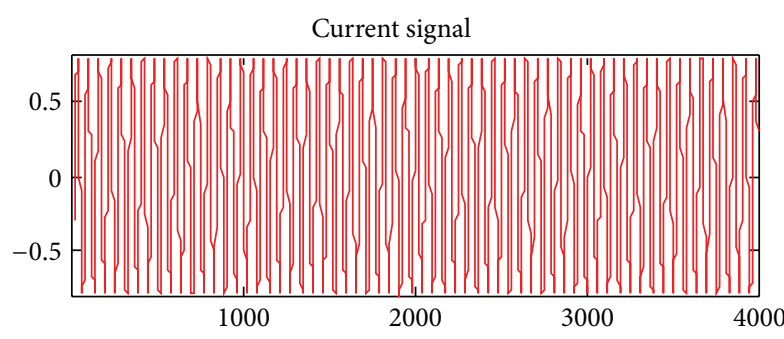

(a)

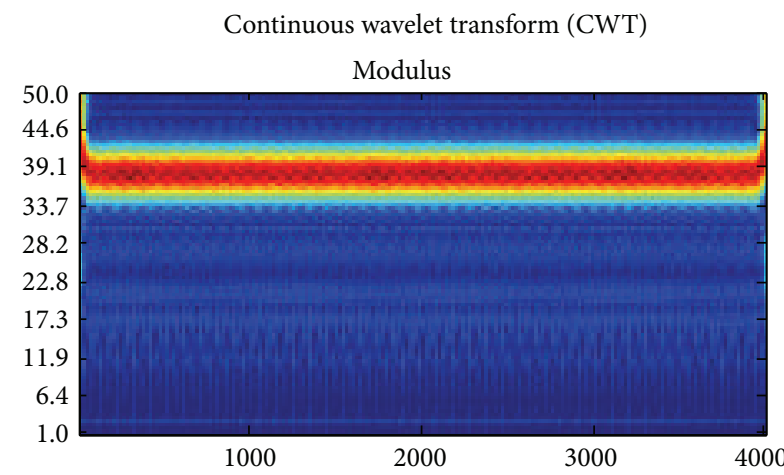

(c)

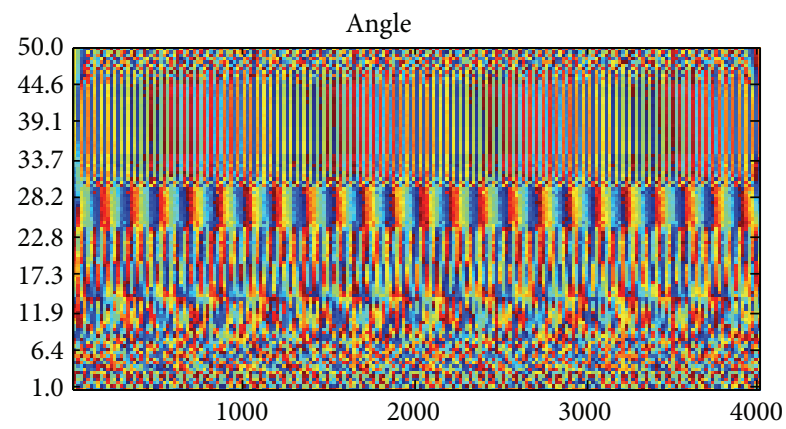

(e)

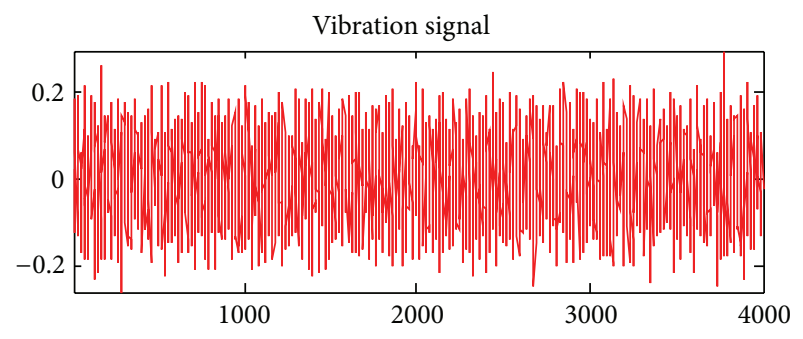

(b)

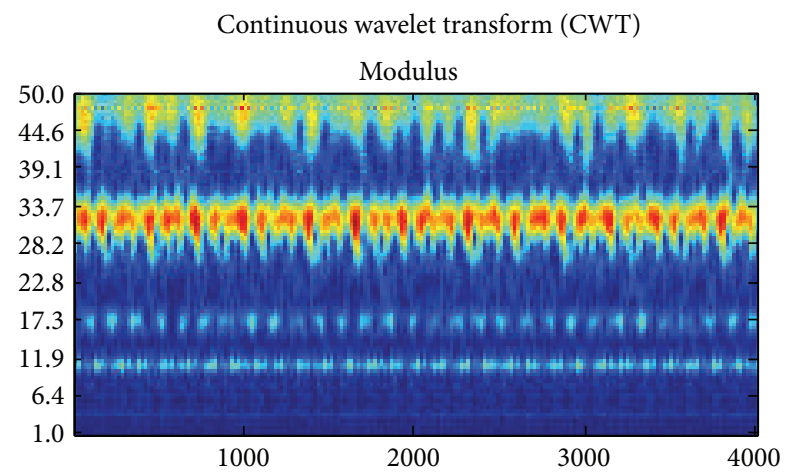

(d)

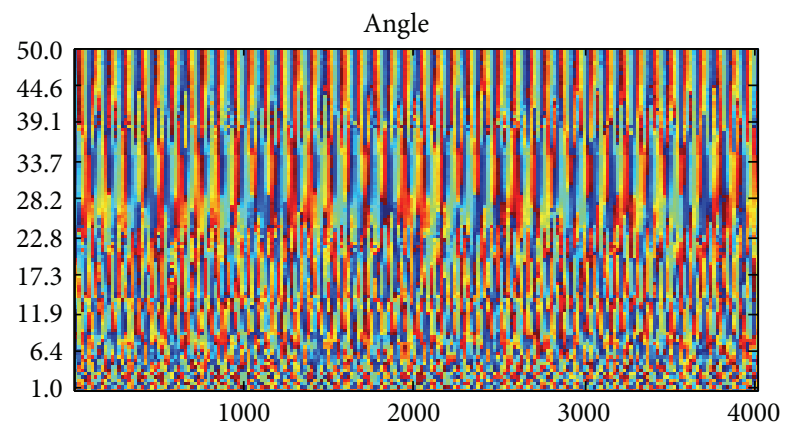

(f)

FIGURE 5: Continuous wavelet transform of DCS and DVS signals.

the two signals in the time-scale plane, consider the wavelet cross spectrum $C_{x y}(a, b)$, which is defined as

$$
C_{x y}(a, b)=\overline{C_{x}(a, b)} C_{y}(a, b) .
$$

The magnitude of the wavelet cross spectrum can be interpreted as the absolute value of the local covariance between the two time series in the time-scale plane, as shown in Figure 6. In this example, this nonnormalized quantity highlights the fact that both signals have a significant contribution around scales 32,16 at all positions.

Figure 7 displays wavelet coherence and the empirical wavelet coherence for $x$ and $y$ is defined as the following ratio:

$$
\frac{s\left(C_{x y}(a, b)\right)}{\sqrt{S\left(\left|C_{x}(a, b)\right|^{2}\right)} \sqrt{S\left(\left|C_{x}(a, b)\right|^{2}\right)}},
$$

where $S$ is a smoothing operator in time and scale. The wavelet coherence can be interpreted as the square of the local correlation coefficient in the time-scale plane.

The common period of the signals at scale 192 is clearly detected using Freq = scal2frq (192, "mother wavelet", 1/sample frequency); note that this corresponds to a frequency of $50 \mathrm{~Hz}$.

The arrows in the figure represent the relative phase between the two signals as a function of scale and position. The relative phase information is obtained from the smoothed estimate of the wavelet cross spectrum, $S\left(C_{x y}(a, b)\right)$. The plot of the relative phases is superimposed on the wavelet coherence. The relative phase information produces a local measure of the delay between the two time series. Note that for scales around 16,28 , and 32 , the direction of the arrows captures the relative phase difference between the two signals. 


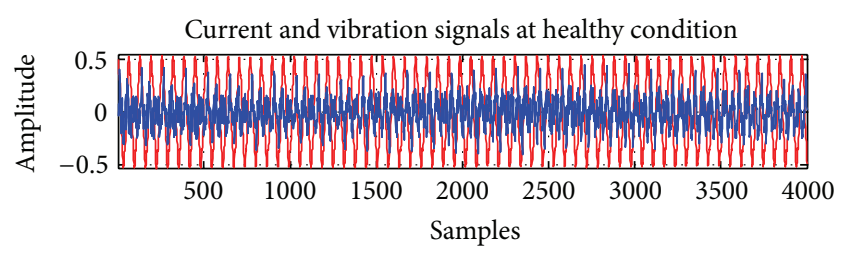

(a)

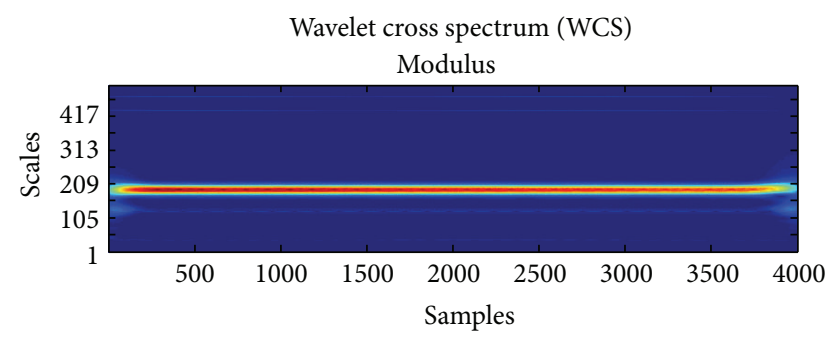

(b)

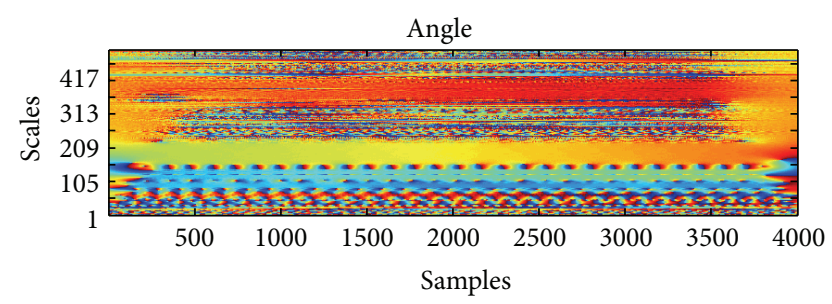

(c)

FIGURE 6: Wavelet cross spectrum of DCS and DVS signals.

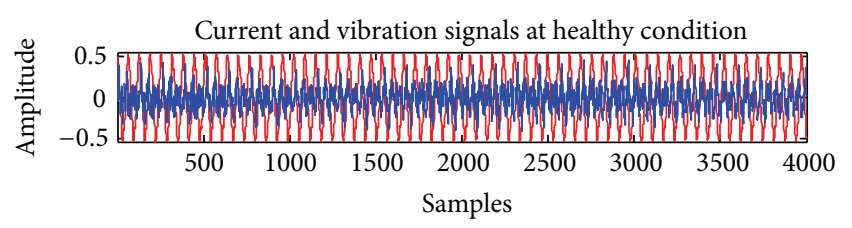

(a)

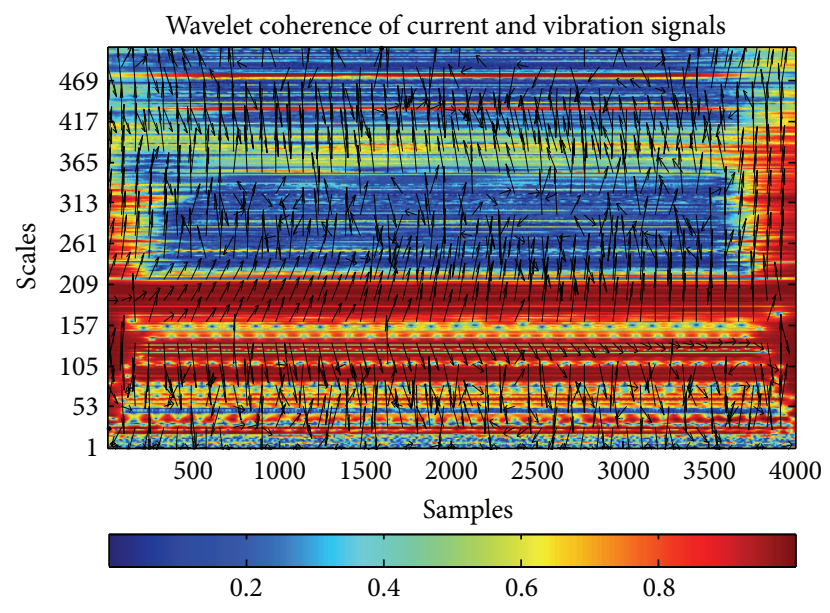

(b)

FIGURE 7: Wavelet coherence of DCS and DVS signals.

\section{Conclusions}

This study utilized Welch's method and continuous wavelet techniques for spectral estimation to investigate the coherence between the driver's current DCS and the driver's vibration signatures. The coherence between DCS and DVS signals was investigated at a particular frequency and in different frequency bands. Both signals are completely coherent if the magnitude-squared coherence is equal to 1 ; if MSC is equal to zero, then both signals are independent of each other. The results show that both signals are coherent at the frequencies at which the magnitude-squared coherence (DSC) is greater than 0.5 and both signals are incoherent (less coherent) if DSC is less than 0.5. Wavelet coherence analysis greatly facilitates the detection of the quasiperiodic component indicative of a system anomaly. Wavelet cross spectrum and wavelet coherence are useful to reveal localized similarities between DCS and DVS signals in the time-scale plane and to interpret the results. The results of this work show the possibility to estimate the DVS signal information from the DCS signal.

\section{References}

[1] B. K. N. Rao, Handbook of Condition Monitoring, Elsevier, Oxford, UK, 1996.

[2] P. A. Higgs, R. Parkin, M. Jackson et al., "A survey on condition monitoring systems in industry," in Proceedings of the 7th Biennial ASME Conference Engineering Systems Design and Analysis, Manchester, UK, 2004.

[3] G. Rohlfing, "Condition monitoring of multiphase pumps," World Pumps, vol. 2010, no. 4, pp. 34-39, 2010.

[4] A. Azadeh, V. Ebrahimipour, and P. Bavar, "A fuzzy inference system for pump failure diagnosis to improve maintenance process: the case of a petrochemical industry," Expert Systems with Applications, vol. 37, no. 1, pp. 627-639, 2010.

[5] R. Ocampo, "Fatigue failures in pumps: part 1," World Pumps, vol. 2008, no. 500, pp. 42-45, 2008.

[6] R. Ocampo and B. Ruiz, "Fatigue failures in pumps: part 2," World Pumps, vol. 2008, no. 502, pp. 18-21, 2008.

[7] C. Hansford, "Condition monitoring: combating down time with vibration sensors," World Pumps, no. 428, pp. 50-53, 2002 2002.

[8] A. Püttmer, "New applications for ultrasonic sensors in process industries," Ultrasonics, vol. 44, supplement 1, pp. e1379-e1383, 2006.

[9] X. Shi, J. Shao, J. Si, and B. Li, "Experiment and simulation of rotor's torsional vibration based on sensorless detection technology," in Proceedings of the IEEE International Conference on Automation and Logistics, pp. 2673-22678, 2008.

[10] C. M. Riley, K. Lin Brian, and T. G. Habetler, "A method for sensorless on-line vibration monitoring of induction machines," IEEE Transactions on Industry Applications, vol. 34, no. 6, pp. 1240-1245, 1998.

[11] S. Seker, E. Ayaz, and E. Türkcan, "Elman's recurrent neural network applications to condition monitoring in nuclear power plant and rotating machinery," Engineering Applications of Artificial Intelligence, vol. 16, no. 7-8, pp. 647-656, 2003. 

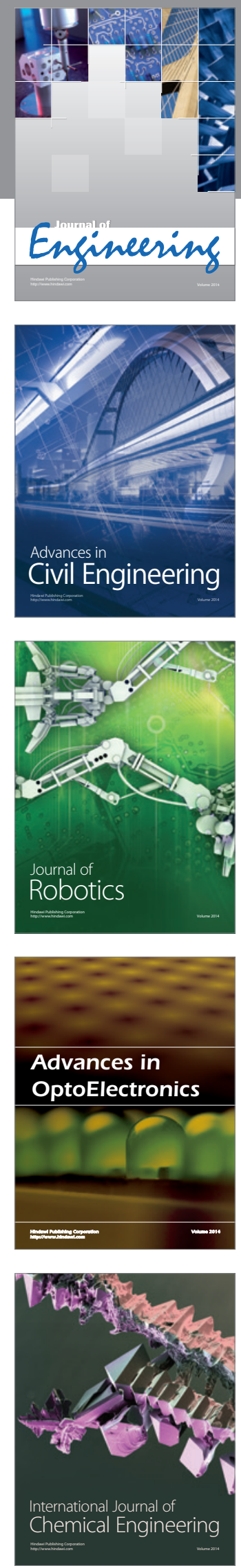

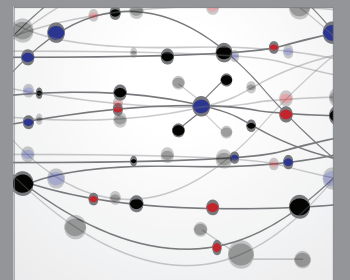

The Scientific World Journal
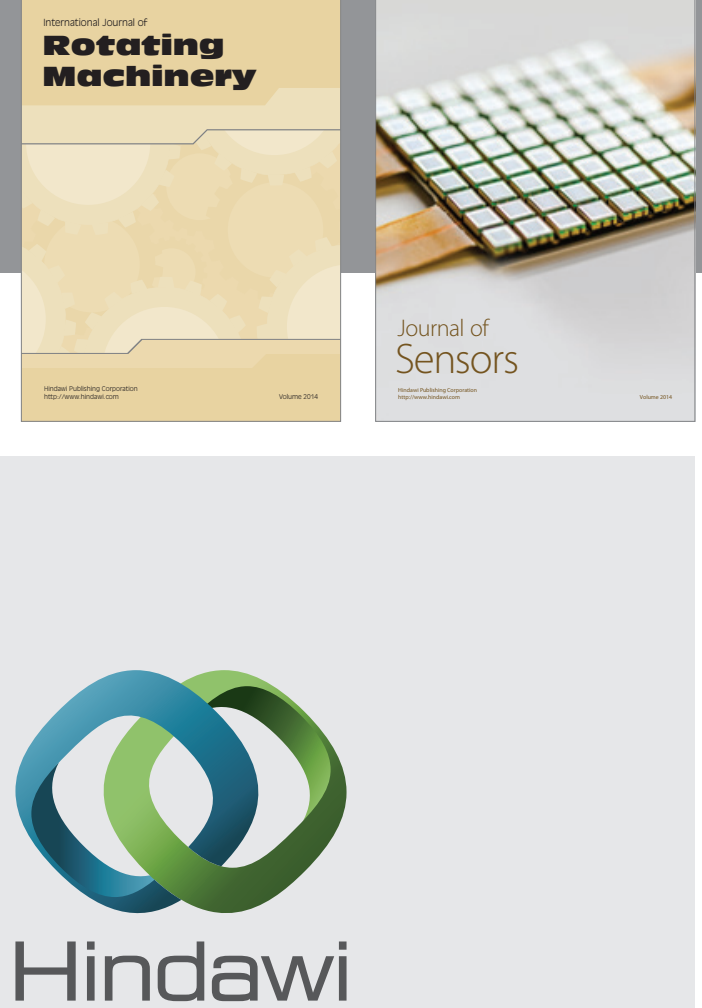

Submit your manuscripts at http://www.hindawi.com
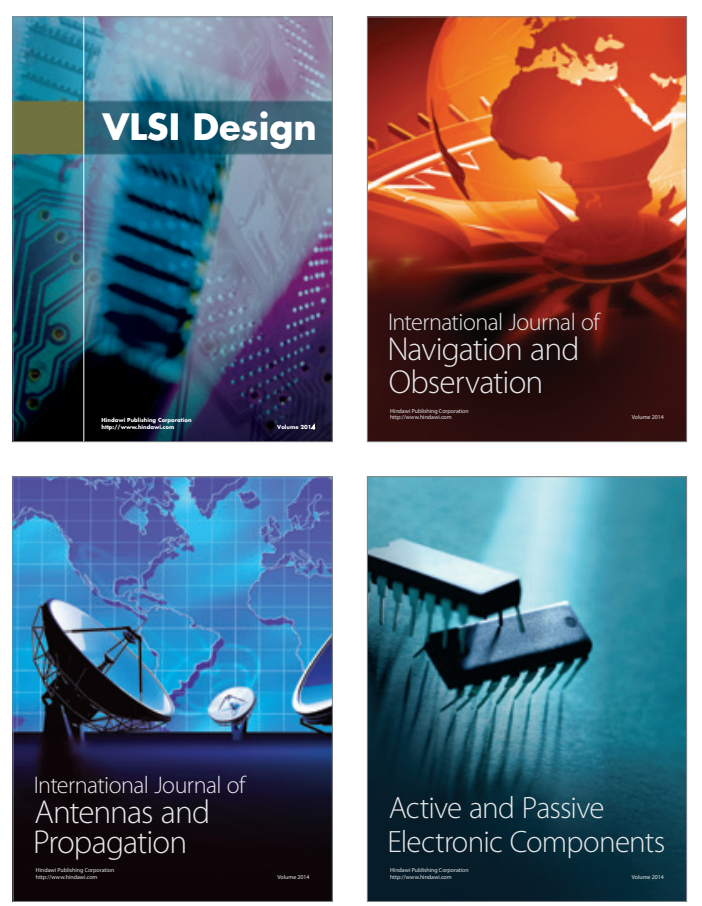
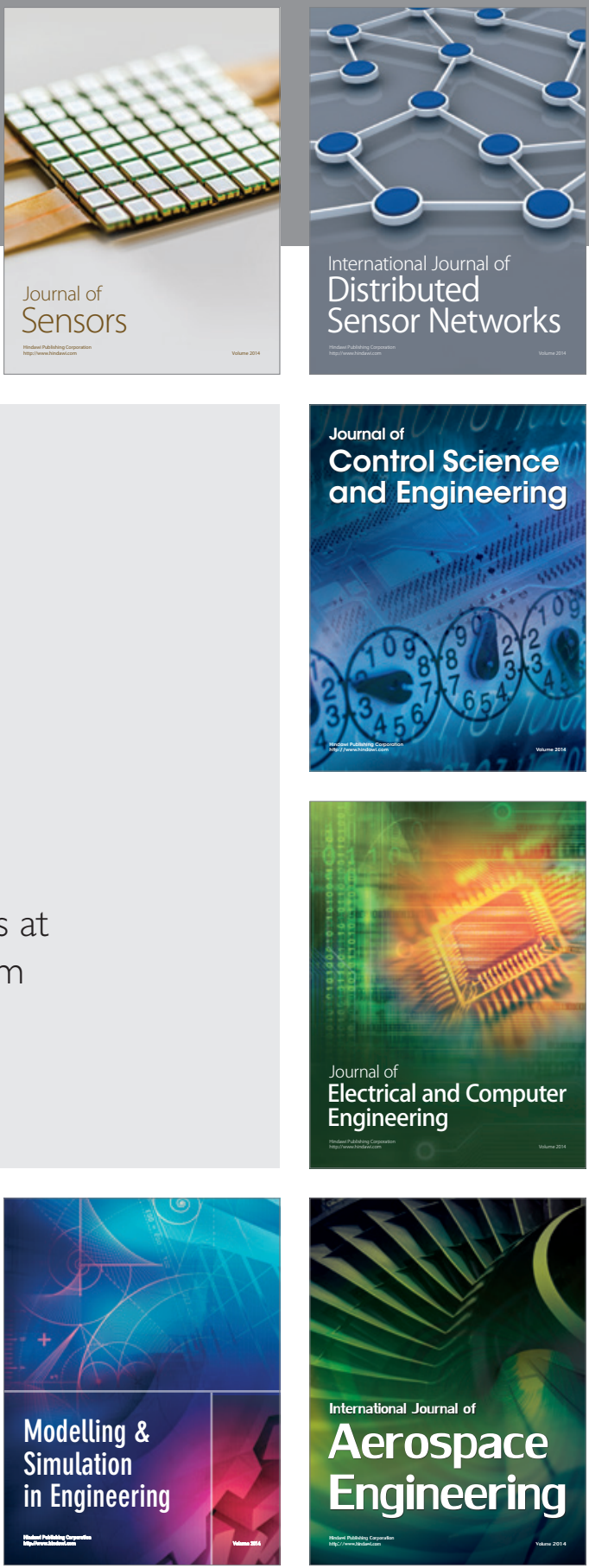

Journal of

Control Science

and Engineering
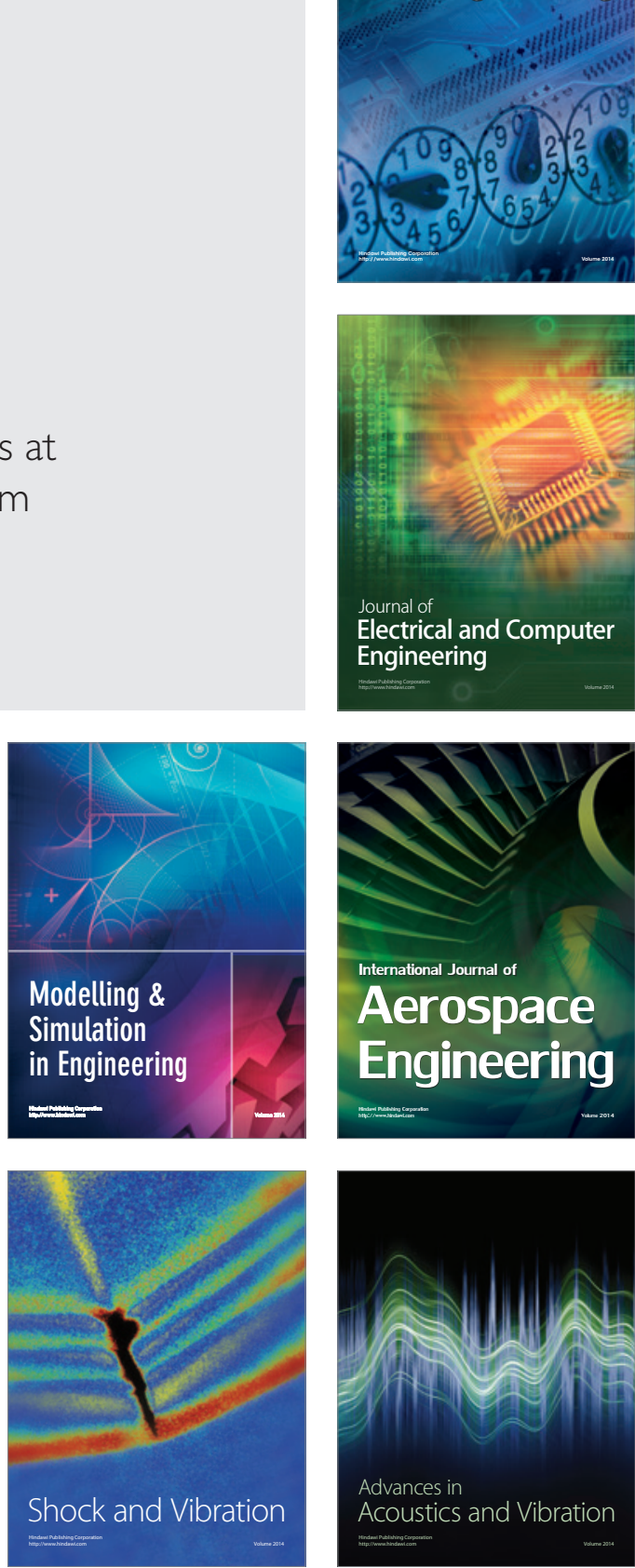